Supporting Information

\title{
Template-Regulated Bimetallic Sulfides Nanozymes with High Specificity and Activity for Visual Colorimetric Detection of Cellular $\mathrm{H}_{2} \mathrm{O}_{2}$
}

Meiling Lian, Meihan Liu, Xiao Zhang, Wei Zhang, Jingbo Zhao, Xiaomeng Zhou* and Da Chen*

Key Laboratory of Civil Aviation Thermal Hazards Prevention and Emergency Response, Civil Aviation University of China, Tianjin 300300, China. 


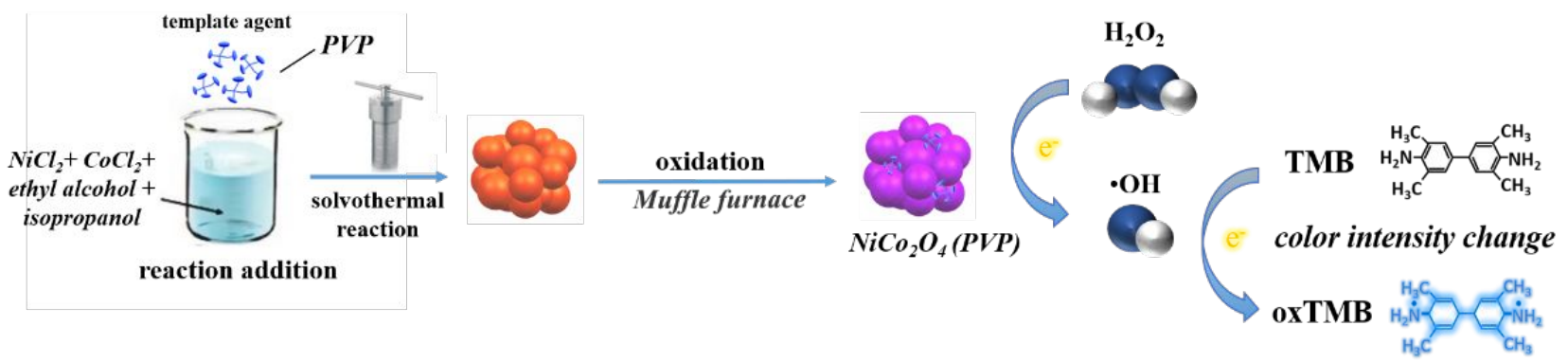

Scheme S1. Schematic illustration of the formation of $\mathrm{NiCo}_{2} \mathrm{O}_{4}$ (PVP) NPs and colorimetric detection of $\mathrm{H}_{2} \mathrm{O}_{2}$. 

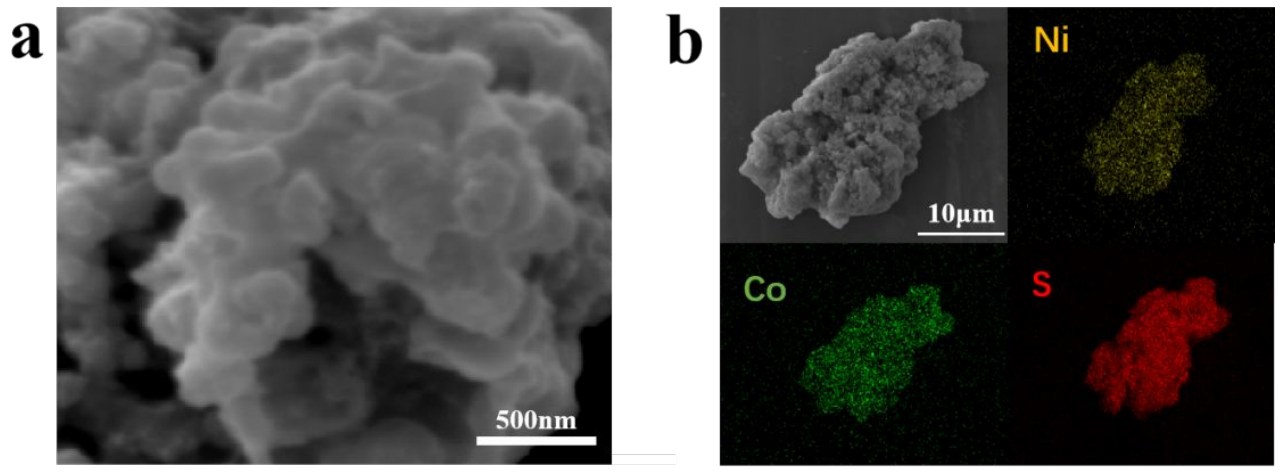

Figure S1. (a) SEM images of $\mathrm{NiCo}_{2} \mathrm{~S}_{4}$ (CTAB) NPs. (b) EDS mapping images of elements of $\mathrm{NiCo}_{2} \mathrm{~S}_{4}(\mathrm{CTAB}) \mathrm{NPs}$. 


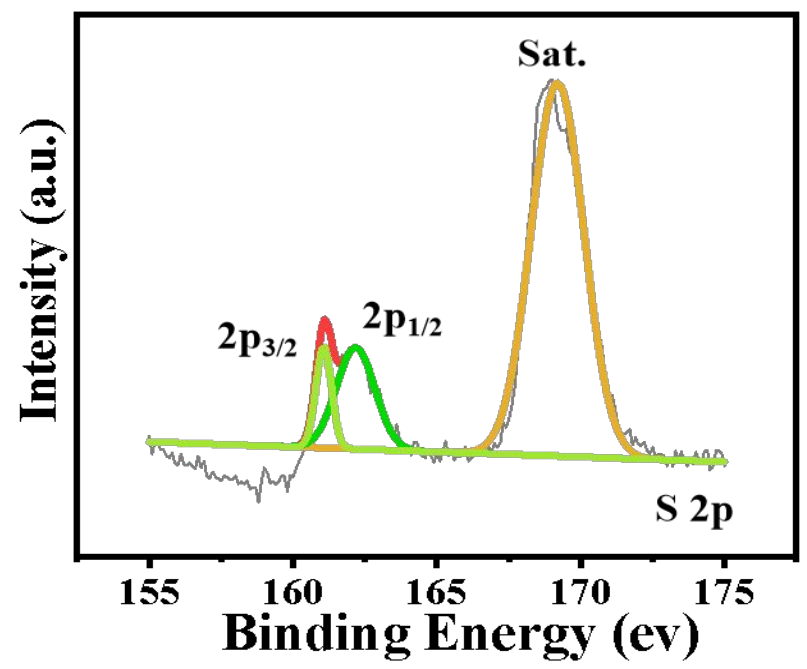

Figure S2. The XPS spectra for $\mathrm{S} 2 \mathrm{p}$ of $\mathrm{NiCo}_{2} \mathrm{~S}_{4}$ (PVP) NPs. 

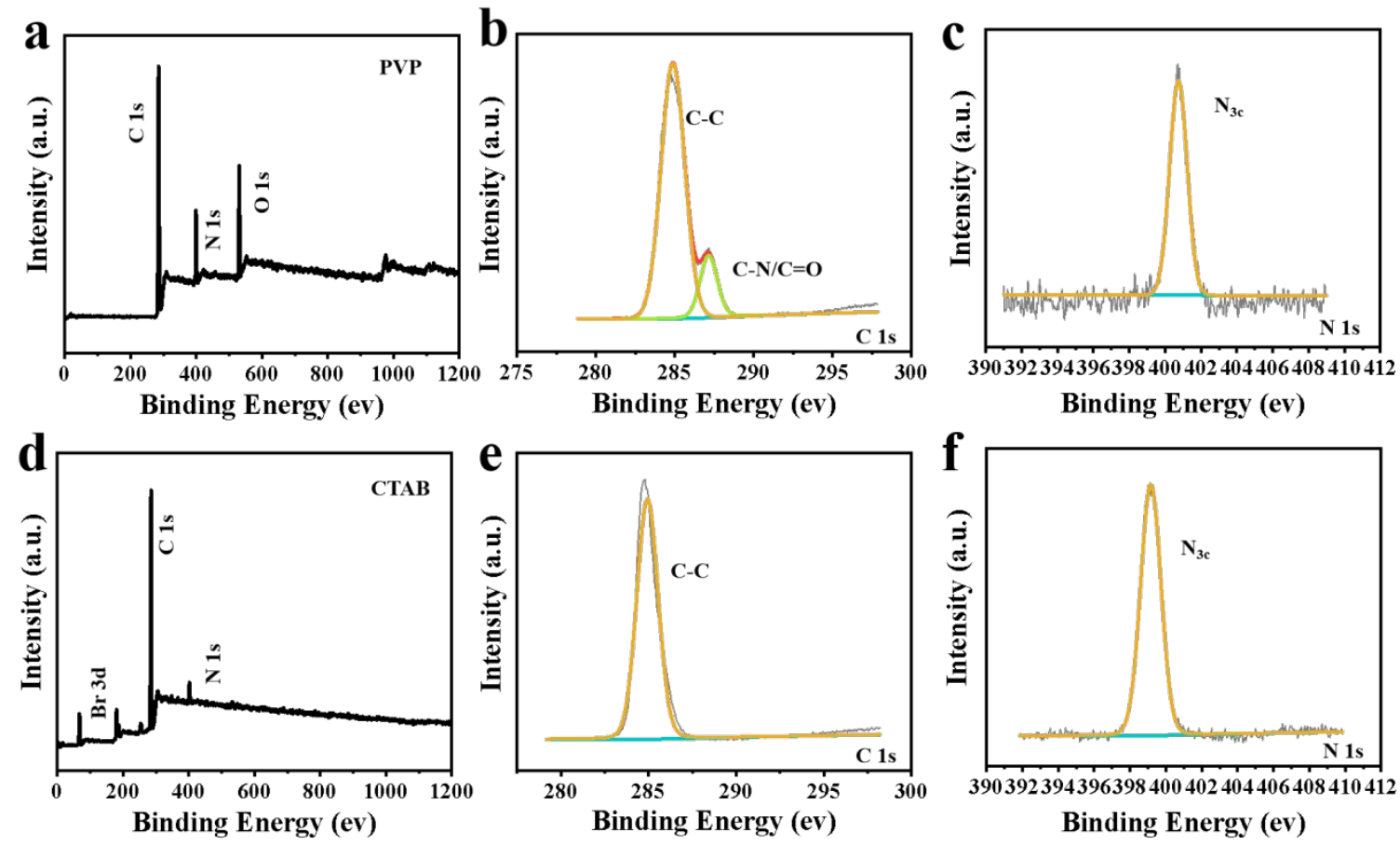

Figure S3. The XPS spectra of (a-c) PVP and (d-f) CTAB. 

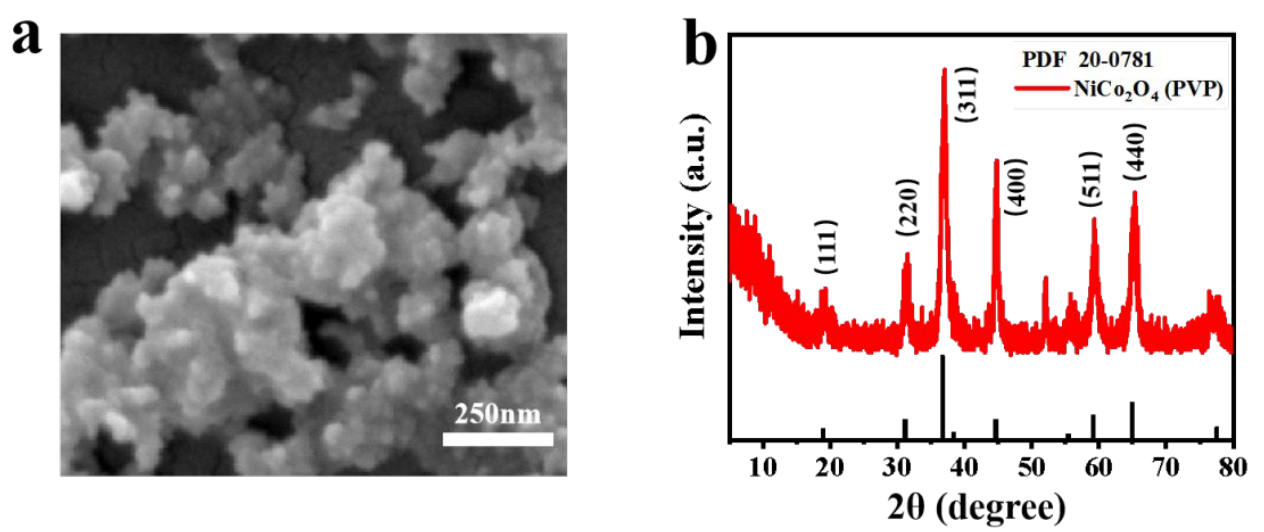

Figure S4. (a) SEM images and (b) XRD of $\mathrm{NiCo}_{2} \mathrm{O}_{4}$ (PVP) NPs. 


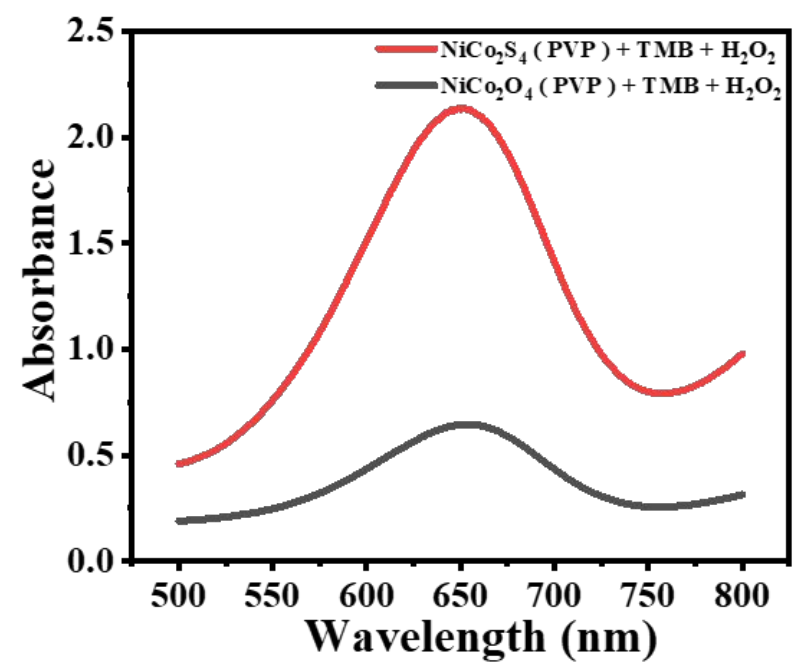

Figure S5. UV-vis absorption spectra of $\mathrm{NiCo}_{2} \mathrm{~S}_{4}(\mathrm{PVP}) \mathrm{NPs}$ and $\mathrm{NiCo}_{2} \mathrm{O}_{4}(\mathrm{PVP}) \mathrm{NPs}$. 


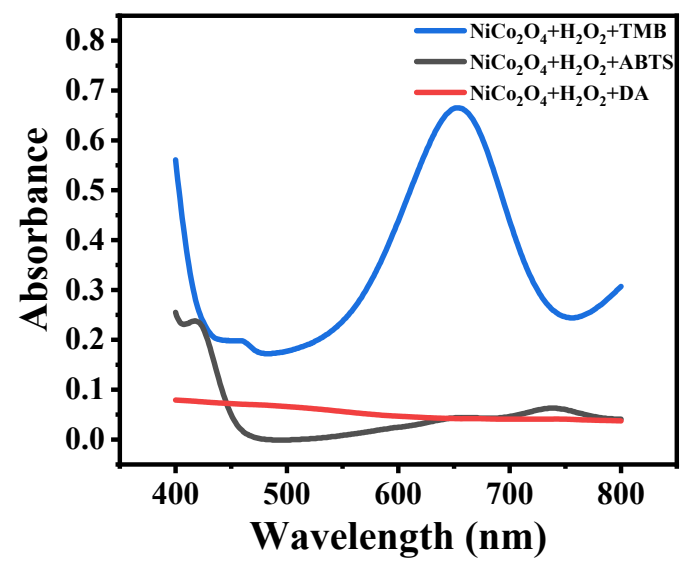

Figure S6. UV-vis absorption spectra of the mixture of $\mathrm{NiCo}_{2} \mathrm{O}_{4}(\mathrm{PVP}) \mathrm{NPs}$ with $100 \mathrm{mM} \mathrm{H}_{2} \mathrm{O}_{2}$ in the presence of various chromogenic substrates (TMB, ABTS, DA). 

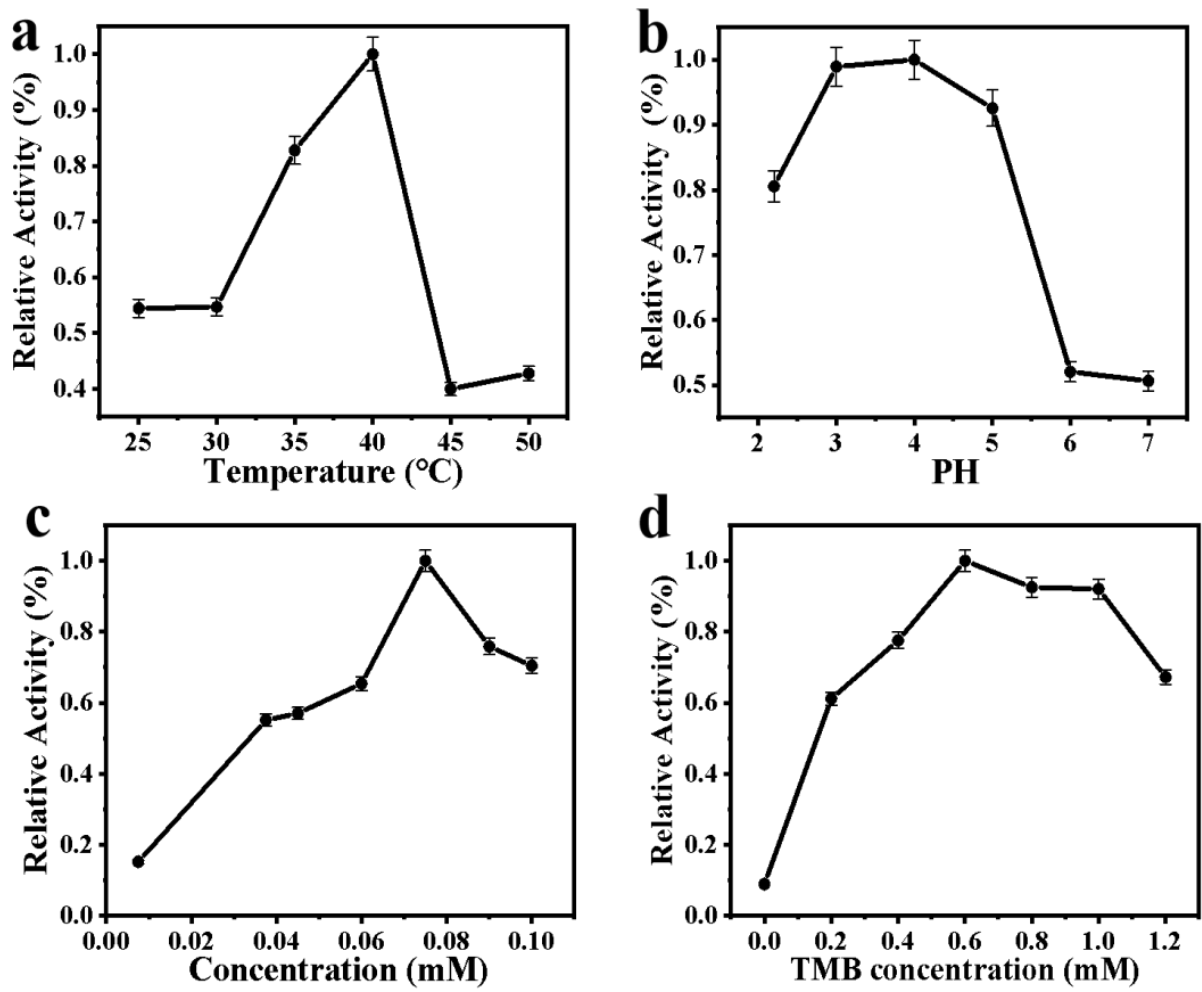

Figure S7. Dependence of the peroxidase-like activity of $\mathrm{NiCo}_{2} \mathrm{~S}_{4}(\mathrm{PVP}) \mathrm{NPs}$ on (a) temperature, (b) $\mathrm{pH}$, (c) concentration and (d) TMB concentration, respectively. 

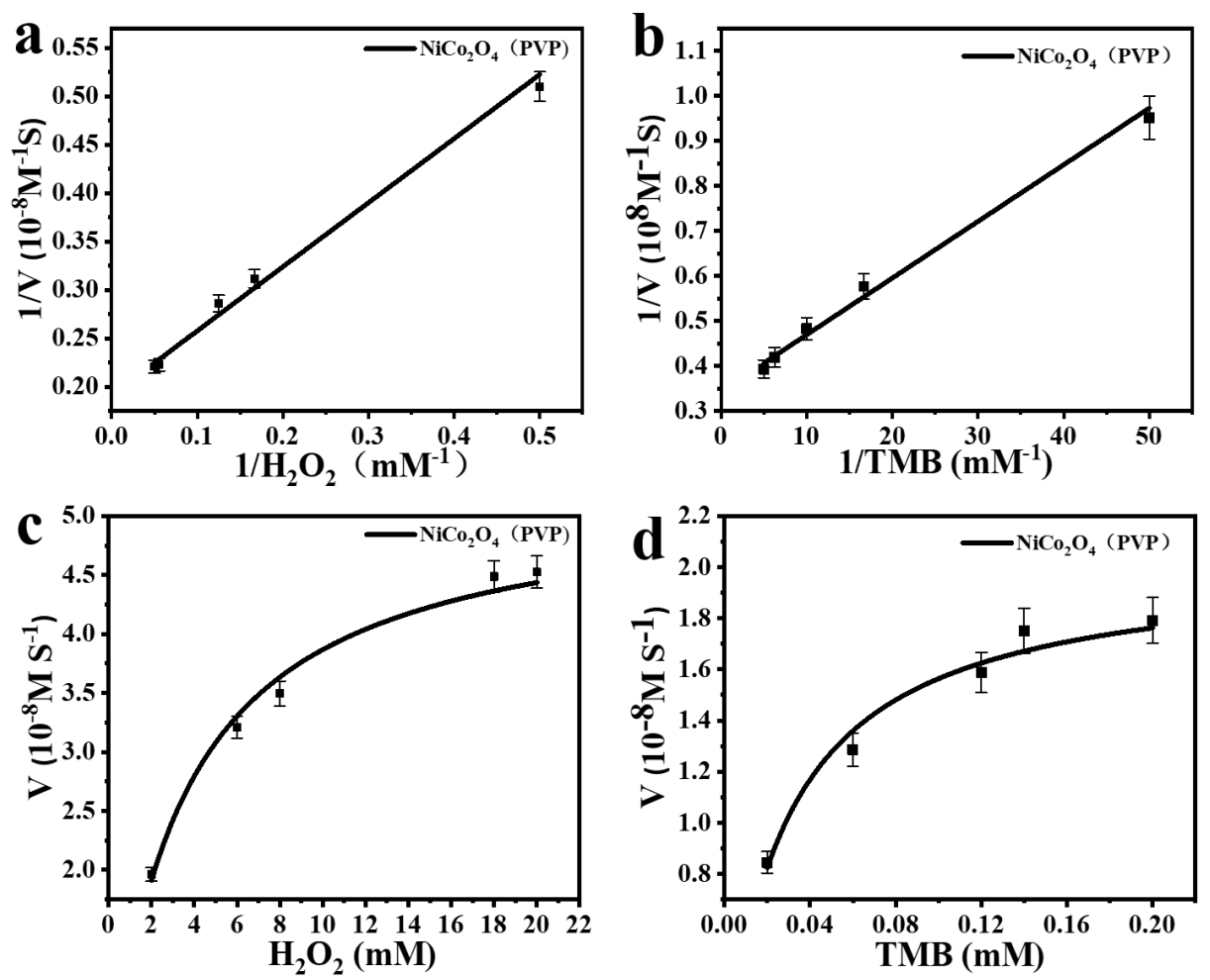

Figure S8. The steady-state kinetic assay of $\mathrm{NiCo}_{2} \mathrm{O}_{4}$ (PVP) NPs. 


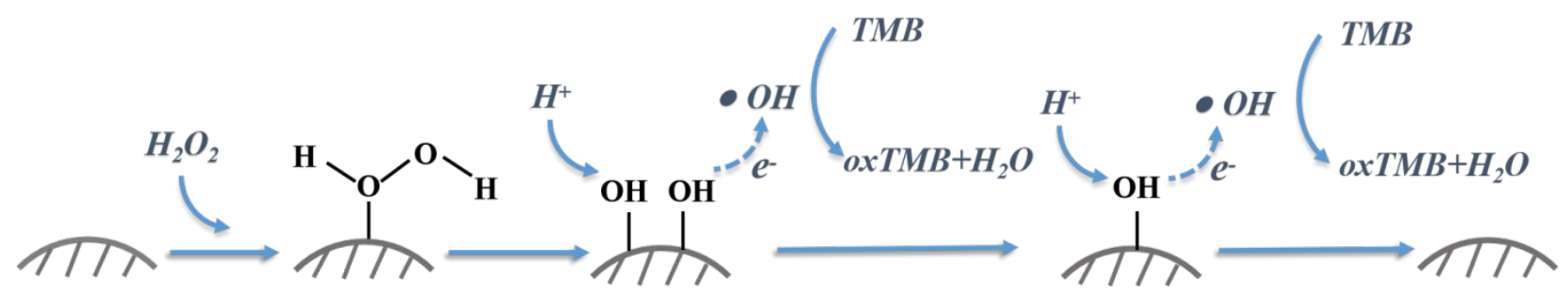

Figure S9. Schematic illustration of the mechanism of the catalytic process and electrons transfer. 

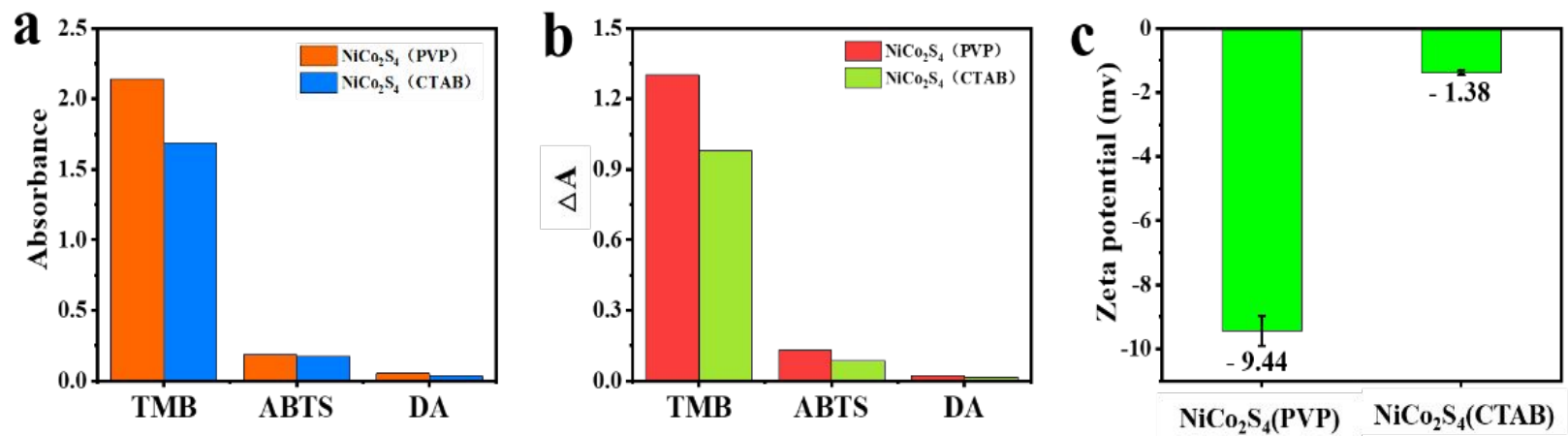

Figure S10. (a) The contrasting colorimetric substrates of $\mathrm{NiCo}_{2} \mathrm{~S}_{4}$ with different template agents (PVP and CTAB) on the surface are selective. (b) The absorbance changes of $\mathrm{NiCo}_{2} \mathrm{~S}_{4}(\mathrm{PVP}) \mathrm{NPs}$ and $\mathrm{NiCO}_{2} \mathrm{~S}_{4}(\mathrm{CTAB}) \mathrm{NPs}$ after adding salt. (c) The zeta potential of $\mathrm{NiCo}_{2} \mathrm{~S}_{4}$ (PVP) NPs and $\mathrm{NiCo}_{2} \mathrm{~S}_{4}(\mathrm{CTAB}) \mathrm{NPs}$. 

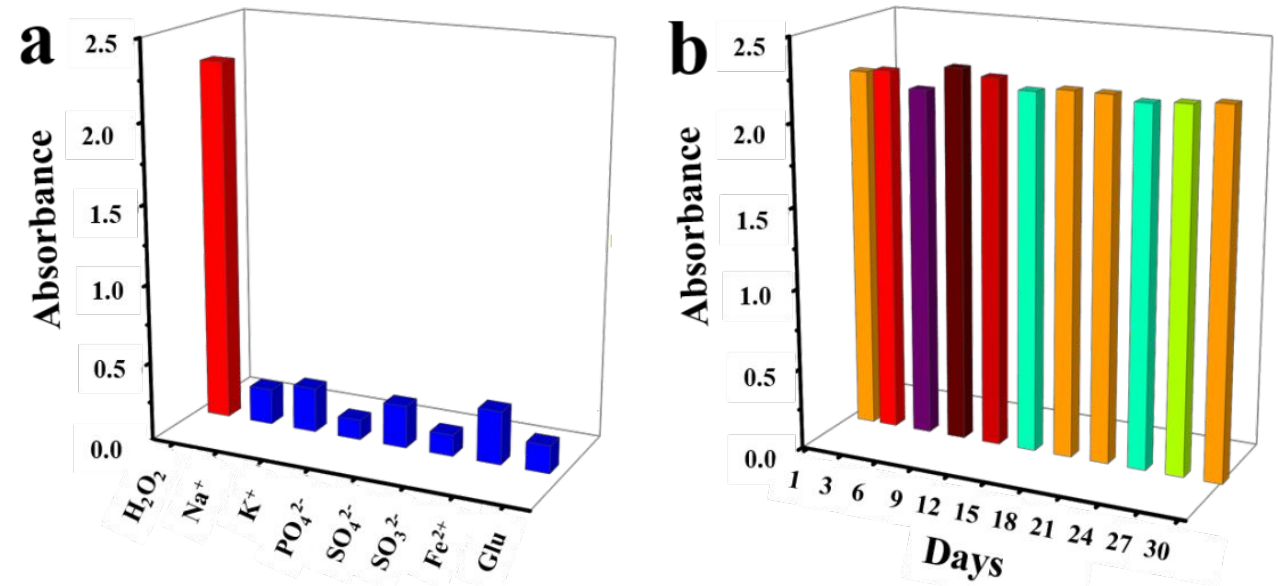

Figure S11. (a) Under the optimal conditions, the selectivity of $\mathrm{H}_{2} \mathrm{O}_{2}$ colorimetric sensor. (b) Stability of $\mathrm{NiCo}_{2} \mathrm{~S}_{4}(\mathrm{PVP}) \mathrm{NPs}$. 
Table S1. Atomic concentration of $\mathrm{Ni}$, $\mathrm{Co}$ and $\mathrm{S}$ in $\mathrm{NiCo}_{2} \mathrm{~S}_{4}(\mathrm{PVP}) \mathrm{NPs}$ and $\mathrm{NiCo}_{2} \mathrm{~S}_{4}(\mathrm{CTAB})$

NPs.

\begin{tabular}{cccc}
\hline Materials & \multicolumn{3}{c}{ Atomic concentration (\%) } \\
\cline { 2 - 4 } & $\mathrm{Ni}$ & $\mathrm{Co}$ & $\mathrm{S}$ \\
\hline $\begin{array}{c}\mathrm{NiCo}_{2} \mathrm{~S}_{4}(\mathrm{PVP}) \\
\mathrm{NPs}\end{array}$ & 3.3 & 10.1 & 10.5 \\
$\mathrm{NiCo}_{2} \mathrm{~S}_{4}(\mathrm{CTAB})$ \\
$\mathrm{NPs}$
\end{tabular}


Table S2. Comparison of Kinetic parameters $\left(\mathrm{K}_{\mathrm{m}}\right.$ and $\left.\mathrm{V}_{\max }\right)$.

\begin{tabular}{cccccc}
\hline Catalyst & \multicolumn{2}{c}{$\mathrm{K}_{\mathrm{m}}(\mathrm{mM})$} & \multicolumn{2}{c}{$\mathrm{V}_{\max }\left(10^{-8} \mathrm{M} \mathrm{s}^{-1}\right)$} & Ref \\
\cline { 2 - 6 } & $\mathrm{TMB}$ & $\mathrm{H}_{2} \mathrm{O}_{2}$ & $\mathrm{TMB}$ & $\mathrm{H}_{2} \mathrm{O}_{2}$ & \\
\hline HRP & 0.43 & 3.70 & 10.00 & 8.71 & {$[1]$} \\
CuS cluster & 0.648 & 29.16 & 5.96 & 4.22 & {$[2]$} \\
FeS NPs & 0.008 & 7.67 & 1.07 & 2.07 & {$[3]$} \\
CoS NPs & 0.41 & 0.26 & 8.45 & 0.61 & {$[4]$} \\
$\mathrm{MoS}_{2} \mathrm{NSs}$ & 0.54 & 2.81 & 3.88 & 8.01 & {$[5]$} \\
$\mathrm{Co} \mathrm{S}_{8}$ & 1.64 & 7.39 & 99.0 & 35.0 & {$[6]$} \\
$\mathrm{NiCo}_{2} \mathrm{~S}_{4}(\mathrm{PVP}) \mathrm{NPs}$ & 0.175 & 7.43 & 7.97 & 19.0 & This work \\
$\mathrm{NiCo}_{2} \mathrm{~S}_{4}(\mathrm{CTAB}) \mathrm{NPs}$ & 0.036 & 6.46 & 3.03 & 9.28 & This work \\
\hline
\end{tabular}


Table S3. Detection performance of $\mathrm{H}_{2} \mathrm{O}_{2}$ in different catalyst systems.

\begin{tabular}{cccc}
\hline Materials & Linear range $(\mathrm{mM})$ & LOD $(\mu \mathrm{M})$ & Ref \\
\hline $\mathrm{Fe}^{2+} / \mathrm{MoO}_{3} \mathrm{NSs}$ & $40-600$ & 11.00 & {$[7]$} \\
$\mathrm{MoS}_{2}-\mathrm{GSSG} \mathrm{NSs}$ & $0.51-50$ & 0.51 & {$[8]$} \\
$\mathrm{FeMnO}_{3} \mathrm{NBs}$ & $0-0.1$ & 3.20 & {$[9]$} \\
$\mathrm{SO}_{4}^{2-} / \mathrm{CoFe}_{2} \mathrm{O}_{4}$ & $0-0.3$ & 6.4 & {$[10]$} \\
$\mathrm{Por}^{-\mathrm{NiCo}_{2} \mathrm{~S}_{4}}$ & $0.02-1.0$ & 10.06 & {$[11]$} \\
$\mathrm{NiCo}_{2} \mathrm{~S}_{4}(\mathrm{PVP})$ & $0.01-0.7$ & 8.00 & This work \\
$\mathrm{NPs}$ & & & \\
\hline
\end{tabular}


Table S4. Detection of $\mathrm{H}_{2} \mathrm{O}_{2}$ in serum.

\begin{tabular}{ccccc}
\hline Sample & Add $(\mu \mathrm{M})$ & Found $(\mu \mathrm{M})$ & Recovery $(\%)$ & RSD $(\%, \mathrm{n}=3)$ \\
\hline Serum & 10 & $10.34 \pm 0.31$ & 103.4 & 5.3 \\
& 50 & $52.4 \pm 1.57$ & 104.8 & 4.7 \\
100 & $99.37 \pm 2.98$ & 99.37 & 5.9 \\
& & & 109.7 & 2.9 \\
\hline
\end{tabular}




\section{References}

(1) Gao, L.-Z.; Zhuang, J.; Nie, L.; Zhang, J.-B.; Zhang, Y.; Gu, N.; Wang, T.-H.; Feng, J.; Yang,

D.-L.; Perrett, S.; Yan, X.-Y.; Intrinsic peroxidase-like activity of ferromagnetic nanoparticles.

Nat. Nanotechnol. 2007, 2, 577-583.

(2) Ding, Y.-N.; Liu, H.; Gao, L.-N.; Fu, M.; Luo, X.-L.; Zhang, X.-X.; Liu, Q.-Y.; Zeng, R.-C.

Fe-doped Ag2S with excellent peroxidase-like activity for colorimetric determination of H2O2. J.

Alloys. Compd. 2019, 785, 1189-1197.

(3) Maji, S. K.; Dutta, A. K.; Biswas, P.; Srivastava, D. N.; Paul, P.; Mondal, A.; Adhikary, B. Synthesis and Characterization of FeS Nanoparticles Obtained from a Dithiocarboxylate Precursor Complex and Their Photocatalytic, Electrocatalytic and Biomimic Peroxidase Behavior. Appl. Catal. A-Gen. 2012, 419-420, 170-177.

(4) Borthakur, P.; Darabdhara, G.; Das, M. R.; Boukherroub, R.; Szunerits, S. Solvothermal Synthesis of CoS/Reduced Porous Graphene Oxide Nanocomposite for Selective Colorimetric Detection of Hg (II) Ion in Aqueous Medium. Sens. Actuator B-Chem. 2017, 244, 684-692.

(5) Yin, W.; Yu, J.; Lv, F.; Yan, L.; Zheng, L.-R.; Gu, Z.; Zhao, Y. Functionalized Nano-MoS2 with Peroxidase Catalytic and Near-Infrared Photothermal Activities for Safe and Synergetic Wound Antibacterial Applications. ACS Nano 2016, 10, 11000-11011.

(6) Borthakur, P.; Darabdhara, G.; R. Das, M.; Boukherroub, R.; Szunerits, S. Solvothermal synthesis of $\mathrm{CoS} /$ reduced porous graphene oxide nanocomposite for selective colorimetric detection of $\mathrm{Hg}$ (II) ion in aqueous medium. Sensor Actuat B-Chem. 2017, 244, 684-692. 
(7) Yang, C.; Xie, Z.-Y.; Zhang, H.-P.; Li, H.; Cai, Y.-H.; Wang, J.-H.; Du, J.-Y.; Yu, H.-L.;

He, Y. Molybdenum Oxide Nanosheet-Supported Ferrous Ion Artificial Peroxidase for Visual Colorimetric Detection of Triacetone Triperoxide. ACS Sustainable Chem. Eng. 2019, 7(23), $18985-18991$.

(8) Yu, J.; Ma, D.-Q.; Mei, L.-Q.; Gao, Q.; Yin, W.-Y.; Zhang, X.; Yan, L.; Gu, Z.-J.; Ma, X.Y.; Zhao, Y.-L. Peroxidase-Like Activity of MoS2 Nanoflakes with Different Modifications and Their Application for H2O2 and Glucose Detection. J. Mater. Chem. B. 2018, 6, 487-498.

(9) Chi, M.-Q.; Chen, S.-H.; Zhong, M.-X.; Wang, C.; Lu, X.-F. Self-Templated Fabrication of FeMnO3 Nanoparticle-Filled Polypyrrole Nanotubes for Peroxidase Mimicking with a Synergistic Effect and Their Sensitive Colorimetric Detection of Glutathione. Chem. Commun. 2018, 54, 5827-5830.

(10) Yin, X.-L.; Liu, P.; Xu, X.-C.; Pan, J.-M.; Li, X.; Niu, X.-H. Breaking the pH limitation of peroxidase-like $\mathrm{CoFe} 2 \mathrm{O} 4$ nanozyme via vitriolization for one-step glucose detection at physiological pH. Sensor Actuat B-Chem. 2021, 328, 129033.

(11) He, Y.-L.; Li, N.; Li, W.-K.; Zhang, X.-X.; Zhang, X.; Liu, Z.-X.; Liu, Q.-Y. 5,10,15,20tetrakis (4-carboxylphenyl) porphyrin functionalized NiCo2S4 yolk-shell nanospheres: Excellent peroxidase-like activity, catalytic mechanism and fast cascade colorimetric biosensor for cholesterol. Sensor Actuat B-Chem. 2021, 326, 128850. 